\title{
Latent variable mixture models to test for differential item functioning: a population- based analysis
}

Xiuyun $\mathrm{Wu}^{1,2}$, Richard Sawatzky ${ }^{3}$, Wilma Hopman ${ }^{4}$, Nancy Mayo ${ }^{5}$, Tolulope T. Sajobi ${ }^{6}$, Juxin Liư ${ }^{7}$, Jerilynn Prior ${ }^{8}$, Alexandra Papaioannou ${ }^{9}$, Robert G. Josse ${ }^{10}$, Tanveer Towheed ${ }^{11}$, K. Shawn Davison ${ }^{12}$ and Lisa M. Lix ${ }^{13^{*}}$

\begin{abstract}
Background: Comparisons of population health status using self-report measures such as the SF-36 rest on the assumption that the measured items have a common interpretation across sub-groups. However, self-report measures may be sensitive to differential item functioning (DIF), which occurs when sub-groups with the same underlying health status have a different probability of item response. This study tested for DIF on the SF-36 physical functioning (PF) and mental health (MH) sub-scales in population-based data using latent variable mixture models (LVMMs).

Methods: Data were from the Canadian Multicentre Osteoporosis Study (CaMos), a prospective national cohort study. LVMMs were applied to the ten PF and five MH SF-36 items. A standard two-parameter graded response model with one latent class was compared to multi-class LVMMs. Multivariable logistic regression models with pseudo-class random draws characterized the latent classes on demographic and health variables.

Results: The CaMos cohort consisted of 9423 respondents. A three-class LVMM fit the PF sub-scale, with class proportions of $0.59,0.24$, and 0.17 . For the $\mathrm{MH}$ sub-scale, a two-class model fit the data, with class proportions of 0 . 69 and 0.31. For PF items, the probabilities of reporting greater limitations were consistently higher in classes 2 and 3 than class 1. For MH items, respondents in class 2 reported more health problems than in class 1. Differences in item thresholds and factor loadings between one-class and multi-class models were observed for both sub-scales. Demographic and health variables were associated with class membership.

Conclusions: This study revealed DIF in population-based SF-36 data; the results suggest that PF and MH sub-scale scores may not be comparable across sub-groups defined by demographic and health status variables, although effects were frequently small to moderate in size. Evaluation of DIF should be a routine step when analysing population-based self-report data to ensure valid comparisons amongst sub-groups.
\end{abstract}

Keywords: Latent class analysis, Item response theory, Mental health, Patient-reported outcome measures, Physical functioning, Population health

\footnotetext{
* Correspondence: lisa.lix@umanitoba.ca

${ }^{13}$ Department of Community Health Sciences, University of Manitoba,

S113-750 Bannatyne Ave, Winnipeg, MB R3E OW3, Canada

Full list of author information is available at the end of the article
} 


\section{Background}

Self-report health status measures, such as the SF-36, are frequently used to compare the health and wellbeing of different populations and to establish population norms for comparative investigations [1]. To make accurate comparisons, the scores on these patientreported outcome measures (PROMs) must be valid and reliable. While many PROMS have been subjected to recommended psychometric evaluations of their construct validity, test-retest reliability, and interpretability [2-4], they are less frequently evaluated for differential item functioning (DIF), a form of measurement nonequivalence. Specifically, measurement non-equivalence (or non-invariance) exists when a PROM does not measure the same construct (e.g., quality of life) across different population sub-groups $[5,6]$. DIF arises when individuals with the same underlying level of health respond differently to the items that comprise a health measure; these differences are often associated with respondent characteristics such as sex and age [7]. If there is no evidence of DIF, individuals with the same characteristics are expected to produce a similar pattern of responses on the PROM items. However, when there is evidence of DIF, differences on the PROM items may be an artifact of the measurement process.

Measurement non-equivalence and DIF have been examined for the SF-36 and SF-12 in population-based data [8-12]. DIF has been detected in both instruments [8, 10-12].

Item response theory (IRT) models, which were originally developed for application to achievement tests in the field of education and psychology, are commonly used to test for DIF [13] amongst manifest (i.e., observed) groups, such as males and females. However, comparisons of item responses for manifest groups using conventional IRT models may lack sensitivity to detect the true source(s) of DIF [14-17]. Specifically, DIF may be associated with unobserved (i.e., latent) characteristics, as well as observed characteristics. Alternative analytic techniques to conventional IRT models are needed to disentangle the effects of latent and observed characteristics [14-17].

Latent variable mixture models (LVMMs) have recently been proposed to detect DIF-related bias in selfreport measures [10, 14-21]. LVMMs combine latent class techniques with IRT; these models do not assume that manifest variables fully account for differential patterns of item functioning [15, 16]. A LVMM for DIF seeks to identify heterogeneous latent groups (i.e., latent classes) in a population with distinct patterns of item responses. The LVMM assumes that the IRT measurement model holds within each latent class, but allows for different sets of item parameters among the latent classes. LVMMs are well suited to the analysis of population- based data, which are likely to exhibit substantial heterogeneity in patterns of item responses. These models are valuable because they can be used to generate hypotheses about population characteristics that may be associated with DIF [10].

The purpose of this study was to test for DIF on selfreport measures of physical and mental health in a diverse population-based cohort using LVMMs. Specifically, we focus on the SF-36 physical functioning (PF) and mental health $(\mathrm{MH})$ sub-scales. These sub-scales were selected for investigation because previous studies have demonstrated their sensitivity to DIF using manifest variable models $[8,9$, $11,22,23]$. An investigation of whether LVMMs will detect DIF in these two SF-36 sub-scales can help to elucidate the causes of DIF and facilitate the interpretation of DIF and its impact on the validity of the SF-36.

\section{Methods}

\section{Data source}

Study data were from the Canadian Multicentre Osteoporosis Study (CaMos), a population-based prospective cohort study that was initiated to provide unbiased national estimates of the prevalence and incidence of osteoporosis [24]. Individuals were recruited without regard for disease status but had to live within a $50-\mathrm{km}$ radius of one of the nine study sites, which were primarily in urban centres. Baseline data, which were the focus of this analysis, were collected in 1996 to 1997 . Details of the data collection methods and sample characteristics have been published elsewhere [24]. We included respondents 25 years of age and older.

\section{Measures}

The SF-36 version 1 was used in the CaMos; it encompasses eight sub-scales: $\mathrm{PF}, \mathrm{MH}$, role physical, bodily pain, general health, vitality, social functioning, and role emotional. The PF sub-scale is comprised of 10 items with three response options: limited a lot, limited a little, and not limited at all. The MH sub-scale is comprised of five items evaluated on a six-point scale with endpoints of all of the time and none of the time [1,25].

The study cohort was described on the demographic variables of sex, age, and education level, and on the health status variables of self-reported general health status, measured height and weight from which body mass index (BMI) was computed, and health utilities. The latter were measured via the Health Utilities Index Mark 3 (HUI3), a preference-based measure that captures functional performance in vision, hearing, speech, ambulation, dexterity, emotion, cognition, and pain [26]. HUI3 summary scores were estimated using a multi-attribute utility function [27]; a score of zero corresponds to death and 1.00 corresponds to a complete absence of impairment. Negative scores are possible and correspond to a severely impaired health state worse than death. 


\section{Statistical Analyses}

Descriptive statistics were used to characterize the study sample on the PF and MH item responses, demographic, and health status measures. Item responses with positively worded formats (i.e., MH3 and MH5) were reverse coded prior to analysis.

Analyses were conducted separately for the PF and $\mathrm{MH}$ sub-scale items. Unidimensionality, the assumption that all items measure a single construct, was investigated by a descriptive analysis of the standardized residuals of item responses for the one-class model [28]. A $\chi^{2}$ statistic was used to test for differences between predicted and observed responses. We also examined unidimensionality using exploratory factor analysis with oblique rotation applied to the polychoric correlations for the items [29]. The root mean square error of approximation (RMSEA) and comparative fit index (CFI) were used to assess model fit. A RMSEA value $\leq 0.10$ [30], and a CFI value $>0.90$ [31] indicate acceptable model fit.

Local independence of the items is satisfied if the residuals of items are not correlated, conditional on the common latent factor (i.e., $P\left[R_{1}, R_{2} \mid \theta\right]=P\left[R_{1} \mid \theta\right] P\left[R_{2} \mid\right.$ $\theta$ ] where $\theta$ represents underlying health status and $R_{1}$ and $R_{2}$ are residuals for scale items 1 and 2. In other words, the joint probability of correct responses for an item pair will be a product of the probabilities of correct responses to the two items, conditional on the latent factor. We evaluated local independence by comparing the predicted and observed proportions of responses for each pair of items in a sub-scale in all the models using a $x^{2}$ statistic [32].

The LVMM combines a latent class model with an IRT two-parameter graded response model (GRM) [33]. This GRM is specified as:

$$
P_{i j\left(X_{i} \geq j \mid \theta, a_{i}\right)}=\frac{\exp \left[\alpha_{i}\left(\theta-\beta_{i j}\right)\right]}{1+\exp \left[\alpha_{i}\left(\theta-\beta_{i j}\right)\right]},
$$

where $P_{i j}$ is the cumulative probability that a person receives a score on the $j$ th or higher category $(j=1,2, \ldots, f)$ for item $i(i=1, \ldots, I)$. In Eq. $1, \theta$ represents underlying health status, $\beta_{i j}$ is the item response parameter (also referred to as the difficulty parameter) for category $j$ or above relative to lower categories and $\alpha_{i}$ is the item discrimination parameter (i.e., slope) for item $i$ indicating the relationship between the item and the latent construct [33].

We adopted Muthén's LVMM framework [34], which estimates factor loadings $(\lambda)$ and item thresholds $(\tau)$; these can be converted to item discrimination and difficulty parameters [35]. The item factor loadings and thresholds in the LVMM are allowed to vary across two or more latent classes (see Figure in Appendix).
The conditional cumulative probability of the item response within the $m$ th latent class is estimated by.

$$
P_{i j m\left(X_{i} \geq j \mid \theta, C=m\right)}=\frac{\exp \left(\lambda_{i m} \theta-\tau_{i j m}\right)}{1+\exp \left(\lambda_{i m} \theta-\tau_{i j m}\right)}
$$

In Eq. 2, $C$ denotes the latent class variable $(m=1, \ldots$, $M), \lambda_{i m}$ is the estimated factor loading for item $i$ within class $m$, and $\tau_{i j m}$ is the estimated threshold for response categories at or above category $j$ for item $i$ within class $m$. The cumulative probability of an item response for category $j$ and higher for a respondent is estimated by:

$$
P_{i j}=\sum_{m=1}^{M}\left(\pi_{m} \times P_{i j m}\left(X_{i} \geq j \mid \theta\right)\right)
$$

where $\pi_{m}$ is the posterior probability for a respondent belonging to the $m$ th latent class [35]. The posterior probability was estimated using Bayes' theorem [36]. Respondents were assigned to the latent class with the largest posterior probability among the classes.

To examine DIF and its impact on the underlying latent health variable (i.e., $\mathrm{PF}$ or $\mathrm{MH}$ ), we adopted a fourstep procedure [10]: (a) fit the standard IRT twoparameter GRM (i.e., one-class model), and multipleclass LVMMs to identify the best-fit model, (b) test for differences in the model parameters across the latent classes, (c) characterize potential sources of DIF by comparing within-class item response percentages and test associations of class membership with the covariates, and (d) examine the impact of DIF by comparing predicted factor scores between the one-class GRM and the multiple-class LVMM.

A one-class model was compared to two-, three-, and four-class models to determine the optimal number of latent classes. The statistics used to select the best-fit model included the Bayesian Information Criterion (BIC) [37, 38], Vuong-Lo-Mendell-Rubin likelihood ratio test (VLMR) [39] and bootstrap likelihood ratio test (BLRT) [37]. A good-fitting multi-class model with estimated class-specific item parameters provides evidence of latent DIF [20]. Quality of class assignment was evaluated with the entropy measure, which ranges between 0 and 1; larger values indicate a higher proportion of correct classifications [40]. The maximum likelihood method with robust standard errors was used to estimate the model parameters [29]. In order to identify the model, the latent factor mean was set to zero and the variance was set to one across classes [34], which is in accordance with conventional IRT parameterization for DIF [34, 41, 42]. The model allowed the item parameters to vary across latent classes. 
The differences in threshold parameters amongst the latent classes were tested for each item using a likelihood ratio (LR) test $[37,43]$. Specifically, a model with free threshold parameters for an item was compared with a model in which the threshold parameters were constrained to be equal across classes for an item. A statistically significant difference between the two models indicates uniform DIF for the item [7].

Multinomial logistic regression with pseudo-class random draws was used to characterize the latent classes on selected demographic and health status variables. Age was categorized as younger (25-64 years) and older $(65+$ years). BMI was categorized as overweight and obese (BMI $\geq 25.0$ ) versus underweight and normal weight $($ BMI $<25.0)$ [44]. Based on the distribution of the HUI3, scores were categorized as low $(<0.8)$ and high $(\geq 0.8)$. Odds ratios (ORs) and 95\% confidence intervals (95\% CIs) were reported.

Predicted factor scores and the most likely class membership for each respondent were obtained from the best-fitting LVMM. The reliability of the predicted factor scores was evaluated by the conditional standard error of measurement (CSEM), which was calculated as the inverse of the square root of the information function [45]. Skewness was examined for the distribution of the predicted factor scores [46].
SAS 9.3 was used to prepare the data and conduct the descriptive analyses [47]. The LVMM analysis was conducted with Mplus version 7.11 [29].

\section{Results \\ Description of cohort}

The CaMos cohort was comprised of 9423 respondents. In total, 9337 (99.1\%), and 9395 respondents (99.7\%) had complete data on the $10 \mathrm{PF}$ items and five $\mathrm{MH}$ items, respectively.

For the PF items, close to half (43.9\%) of respondents reported being limited a lot in vigorous activities, while less than $6.0 \%$ of respondents reported being limited a lot when climbing one flight of stairs, walking one block, or bathing/dressing (Table 1). For the $\mathrm{MH}$ items, less than $2.0 \%$ of respondents reported that they were nervous, so down in the dumps that nothing could cheer them up, or feeling downhearted and blue all of the time.

\section{Examination of unidimensionality and local independence} For the PF sub-scale items, the IRT two-parameter GRM fit the data well as evidenced by absolute values of standardized residuals less than 1.96 for all items. The difference between observed and predicted proportions of item responses was not statistically significant

Table 1 Distribution of item responses (\%) for the SF-36 sub-scale items

\begin{tabular}{|c|c|c|c|c|c|c|}
\hline \multirow{2}{*}{$\begin{array}{l}\text { Item } \\
\text { Physical Functioning ( } N=9337)\end{array}$} & \multicolumn{6}{|c|}{ Response option } \\
\hline & Limited a lot & \multicolumn{2}{|c|}{ Limited a little } & \multicolumn{3}{|c|}{ Not limited at all } \\
\hline PF1: Vigorous activities & 43.9 & \multicolumn{2}{|l|}{34.2} & \multicolumn{3}{|l|}{21.9} \\
\hline PF2: Moderate activities & 12.7 & \multicolumn{2}{|l|}{24.4} & \multicolumn{3}{|l|}{62.9} \\
\hline PF3: Lifting or carrying groceries & 9.1 & \multicolumn{2}{|l|}{22.2} & \multicolumn{3}{|l|}{68.8} \\
\hline PF4: Climbing several flights of stairs & 15.2 & \multicolumn{2}{|l|}{29.6} & \multicolumn{3}{|l|}{55.1} \\
\hline PF5: Climbing one flight of stairs & 5.6 & \multicolumn{2}{|l|}{15.8} & \multicolumn{3}{|l|}{78.6} \\
\hline PF6: Bending, kneeling or stooping & 13.0 & \multicolumn{2}{|l|}{33.0} & \multicolumn{3}{|l|}{54.0} \\
\hline PF7: Walking more than a mile & 16.5 & \multicolumn{2}{|l|}{20.6} & \multicolumn{3}{|l|}{62.9} \\
\hline PF8: Walking several blocks & 10.8 & \multicolumn{2}{|l|}{15.0} & \multicolumn{3}{|l|}{74.2} \\
\hline PF9: Walking one block & 4.0 & \multicolumn{2}{|l|}{9.9} & \multicolumn{3}{|l|}{86.0} \\
\hline PF10: Bathing or dressing self & 2.0 & \multicolumn{2}{|l|}{6.5} & \multicolumn{3}{|l|}{91.5} \\
\hline Mental Health $(N=9395)$ & $\begin{array}{l}\text { All of } \\
\text { the time }\end{array}$ & $\begin{array}{l}\text { Most } \\
\text { of the time }\end{array}$ & $\begin{array}{l}\text { Good bit } \\
\text { of the time }\end{array}$ & $\begin{array}{l}\text { Some } \\
\text { of the time }\end{array}$ & $\begin{array}{l}\text { Little } \\
\text { of the time }\end{array}$ & $\begin{array}{l}\text { None } \\
\text { of the time }\end{array}$ \\
\hline \multicolumn{7}{|l|}{ Have you.. } \\
\hline MH1: Been a very nervous person? & 1.3 & 3.1 & 5.4 & 17.2 & 29.7 & 43.3 \\
\hline $\begin{array}{l}\mathrm{MH} \text { 2: Felt so down in the dumps that } \\
\text { nothing could cheer you up? }\end{array}$ & 0.3 & 1.1 & 1.8 & 7.4 & 17.0 & 72.4 \\
\hline MH3: Felt calm and peaceful? & 10.6 & 46.3 & 18.2 & 15.7 & 5.9 & 3.2 \\
\hline MH4: Felt downhearted and blue? & 0.4 & 1.6 & 3.4 & 17.8 & 34.8 & 42.1 \\
\hline MH5: Been a happy person? & 18.5 & 54.8 & 12.9 & 8.7 & 2.9 & 2.3 \\
\hline
\end{tabular}


$\left(x^{2}=9.01, \mathrm{df}=27, p=0.999\right)$, suggesting that the 10 items represented a single construct.

Confirmatory factor analysis for a single latent construct resulted in RMSEA $=0.11$ and CFI $=0.98$, with the former indicating poor model fit. A confirmatory factor analysis model with error covariances between five pairs of items (PF1 and PF2, PF2 and PF3, PF4 and PF5, PF7 and PF8, PF8 and PF9) resulted in a good fit (RMSEA $=0.057$, CFI $=0.99$ ) Thus, the factor structure suggested a single latent factor solution for the PF scale items. Pairwise comparisons of predicted and observed proportions of item responses for all items revealed a poor fit to the data $\left(X^{2}=3020.96, \mathrm{df}=351, p<0.001\right)$, suggesting that the assumption of local independence might not be tenable and heterogeneous subgroups might exist in the sample $[16,48]$.

For the MH sub-scale, the IRT two-parameter GRM fit the data well with respect to a unidimensional model. Absolute values of standardized residuals were less than 1.96 for all sub-scale items. Univariate model fit statistics indicated a non-significant difference between observed and predicted item responses $\left(\mathrm{x}^{2}=6.61, \mathrm{df}=29, p=0.999\right)$. Confirmatory factor analysis revealed that one-factor model was not a good fit as judged by the RMSEA $=0.15$, although the CFI (0.97) indicated good fit. These results support a single dominant latent factor for both the PF and $\mathrm{MH}$ sub-scale items. However, a statistically significant difference in the joint distributions of item pairs $(\mathrm{x} 2=1952.12$, $\mathrm{df}=159, p<0.001)$ for $\mathrm{MH}$ sub-scale was also observed, suggesting that local dependence might exist, and thus a mixture IRT model with more than one class might be a better choice to account for local dependence.

\section{Fitting models with latent classes}

For the PF sub-scale, the three-class LVMM had a better fit than the one- and two-class models; the BIC, VLMR and BLRT all favored the three-class model (Table 2). For the MH sub-scale, the two-class model yielded better fit indices than the one-class GRM. Therefore, threeand two-class models were chosen as the best-fitting models for the PF and $\mathrm{MH}$ sub-scales, respectively (Table 2).

For the PF sub-scale items, threshold values in class 2 were uniformly larger than in class 1 . As well, there were larger threshold values amongst respondents in class 3 than in class 2. This indicates that respondents with the same level of latent ability had higher probabilities of endorsing lower categories for items representing limited PF in class 2 and class 3 relative to class 1 . Differences in factor loadings were found for the items across the classes, suggesting that items varied in their ability to differentiate amongst respondents.

For the MH sub-scale, the absolute value of differences in item thresholds between the two classes ranged from 0.38 for MH1 to 3.56 for MH5. Factor loadings for class 2 were smaller than for class 1 .

Table 3 shows the LR statistics to test for DIF on PF and $\mathrm{MH}$ sub-scale items. Overall, nine of the 10 items for the PF sub-scale and all the five items in the $\mathrm{MH}$ sub-scale showed uniform DIF.

\section{Sources of DIF}

Figure 1 compares the response percentages for each of the three latent classes on the PF sub-scale items. The prevalence of PF limitations was significantly higher in classes 2 and 3 compared to class 1 . Overall, $98.2 \%$ of respondents reported having limitations in class 3 on "vigorous activities" compared to only $67.4 \%$ for class 1 .

Table 2 Model fit statistics and class proportions for latent variable mixture models on the SF-36 physical functioning and mental health sub-scale items

\begin{tabular}{|c|c|c|c|c|c|c|c|c|c|}
\hline \multirow{2}{*}{$\begin{array}{l}\# \\
\text { Classes }\end{array}$} & \multirow{2}{*}{$\begin{array}{l}\# \\
\text { Par }\end{array}$} & \multirow[t]{2}{*}{$\mathrm{BIC}$} & \multirow{2}{*}{$\begin{array}{l}\text { VLMR } \\
p \text {-value }\end{array}$} & \multirow{2}{*}{$\begin{array}{l}\text { BLRT } \\
p \text {-value }\end{array}$} & \multirow[t]{2}{*}{ Entropy } & \multicolumn{4}{|c|}{ Class proportions $^{a}$} \\
\hline & & & & & & Class 1 & Class 2 & Class 3 & Class 4 \\
\hline \multicolumn{10}{|c|}{ Physical Functioning } \\
\hline 1 & 28 & $102,298.56$ & & & & 1.00 & & & \\
\hline 2 & 57 & $100,569.46$ & $<0.0001$ & $<0.0001$ & 0.55 & $0.61(0.68)$ & $0.39(0.32)$ & & \\
\hline 3 & 86 & $100,083.64$ & 0.0007 & $<0.0001$ & 0.64 & $0.59(0.64)$ & $0.24(0.22)$ & $0.17(0.14)$ & \\
\hline 4 & 115 & $99,806.68$ & NA & NA & 0.60 & $\begin{array}{l}0.51 \\
(0.55)\end{array}$ & $0.22(0.19)$ & $0.17(0.16)$ & $0.10(0.10)$ \\
\hline \multicolumn{10}{|c|}{ Mental Health } \\
\hline 1 & 20 & $95,536.81$ & & & & 1.00 & & & \\
\hline 2 & 41 & $94,631.65$ & $<0.0001$ & $<0.0001$ & 0.40 & $0.69(0.78)$ & $0.31(0.22)$ & & \\
\hline 3 & 62 & $94,363.51$ & NA & NA & 0.44 & $0.25(0.20)$ & $0.37(0.40)$ & $0.38(0.40)$ & \\
\hline
\end{tabular}

Bold values indicate the model that was selected for further analysis. \# Par = number of parameters. BIC Bayesian information criterion. VLMR Vuong-Lo-MendellRubin likelihood ratio test. BLRT Bootstrap likelihood ratio test. NA Not Applicable

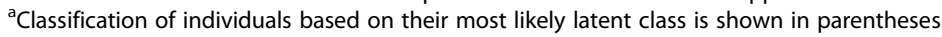


Table 3 Likelihood ratio (LR) test statistics for differential item functioning on the SF-36 physical functioning and mental health subscale items

\begin{tabular}{|c|c|c|c|}
\hline Item & LR Statistic & $\Delta \mathrm{df}^{*}$ & $p$-value \\
\hline \multicolumn{4}{|l|}{ Physical Functioning } \\
\hline PF1: Vigorous activities & 99.63 & 4 & $<0.0001$ \\
\hline PF2: Moderate activities & 127.49 & 4 & $<0.0001$ \\
\hline PF3: Lifting or carrying groceries & 151.04 & 4 & $<0.0001$ \\
\hline PF4: Climbing several flights of stairs & 37.69 & 4 & $<0.0001$ \\
\hline PF5: Climbing one flight of stairs & 60.95 & 4 & $<0.0001$ \\
\hline PF6: Bending, kneeling or stooping & 141.85 & 4 & $<0.0001$ \\
\hline PF7: Walking more than a mile & 87.79 & 4 & $<0.0001$ \\
\hline PF8: Walking several blocks & 34.51 & 4 & $<0.0001$ \\
\hline PF9: Walking one block & 3.26 & 2 & 0.196 \\
\hline PF10: Bathing or dressing self & 64.76 & 2 & $<0.0001$ \\
\hline \multicolumn{4}{|l|}{ Mental Health } \\
\hline MH1: Been a very nervous person? & 71.79 & 3 & $<0.0001$ \\
\hline $\begin{array}{l}\mathrm{MH} 2 \text { : Felt so down in the dumps that } \\
\text { nothing could cheer you up? }\end{array}$ & 21.03 & 3 & 0.0001 \\
\hline MH3: Felt calm and peaceful? & 234.43 & 3 & $<0.0001$ \\
\hline MH4: Felt downhearted and blue? & 52.27 & 3 & $<0.0001$ \\
\hline MH5: Been a happy person? & 198.28 & 3 & $<0.0001$ \\
\hline
\end{tabular}

LR statistics are for the comparison of nested models: (a) mixture IRT model with free thresholds across classes for each item, and (b) IRT model with constrained thresholds across classes for each item. The mixture IRT model accounted for local dependence. Note that the latent variable mixture model for the physical functioning sub-scale had 3 classes and the model for the mental health sub-scale had 2 classes. $\Delta \mathrm{df}$ is the difference in degrees of freedom for models defined in (a) and (b)

As well, $99.8 \%$ of respondents had limitations on "climbing several flights of stairs" in class 3 compared to only $26.7 \%$ in class 1.

Similarly, respondents reported more $\mathrm{MH}$ problems in class 2 than class 1 (Fig. 2). For example, 15.2\% of respondents in class 2 reported having been a very nervous person $(\mathrm{MH} 1)$ all of the time/most of the time/a good bit of the time, compared to just $8.3 \%$ of respondents in class 1 . Close to half $(47.4 \%)$ of respondents in class 2 reported feeling calm and peaceful none of the time/little of the time/some of the time relative to only $18.5 \%$ of respondents in class 1.

Table 4 contains the results for the logistic regression analysis of the associations between latent class membership

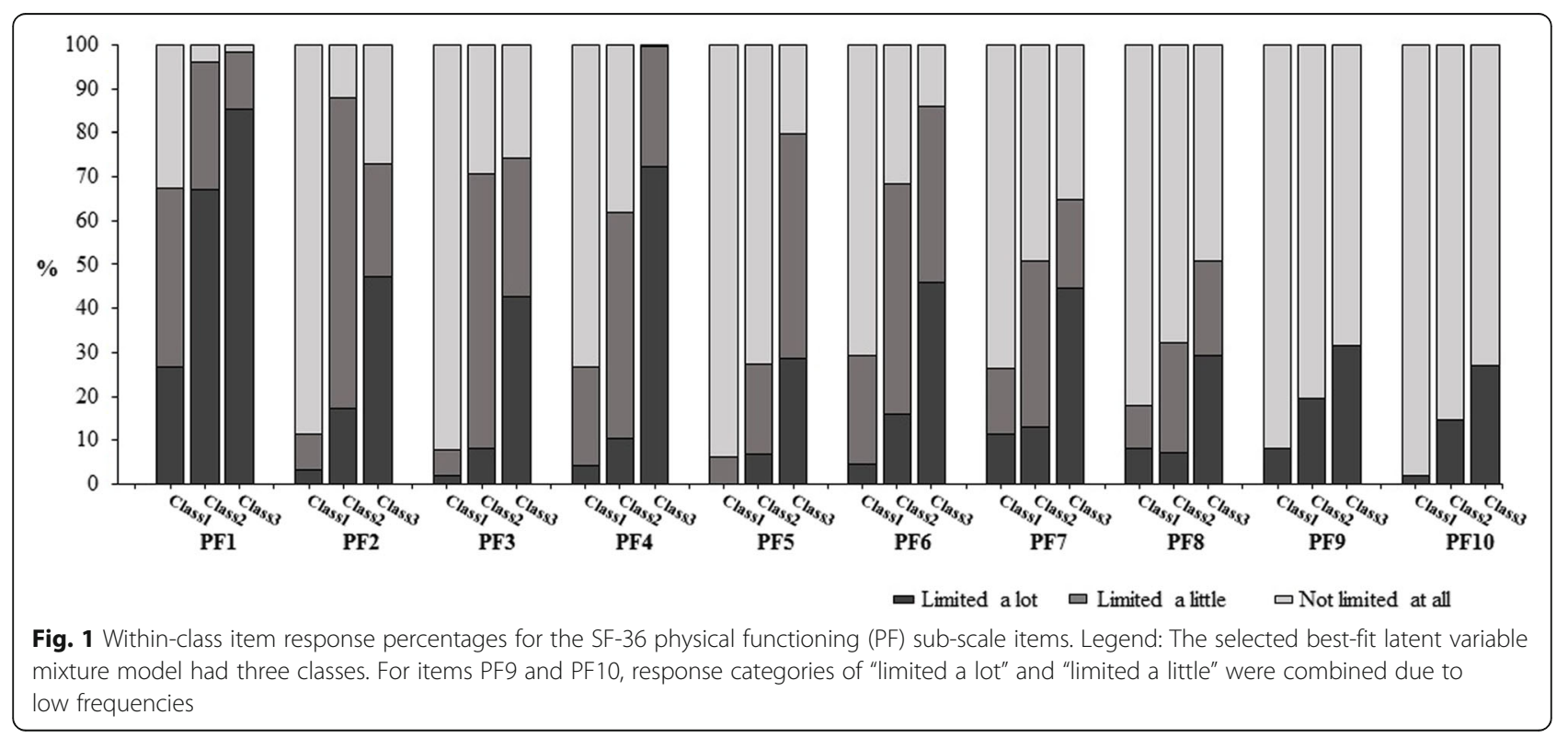




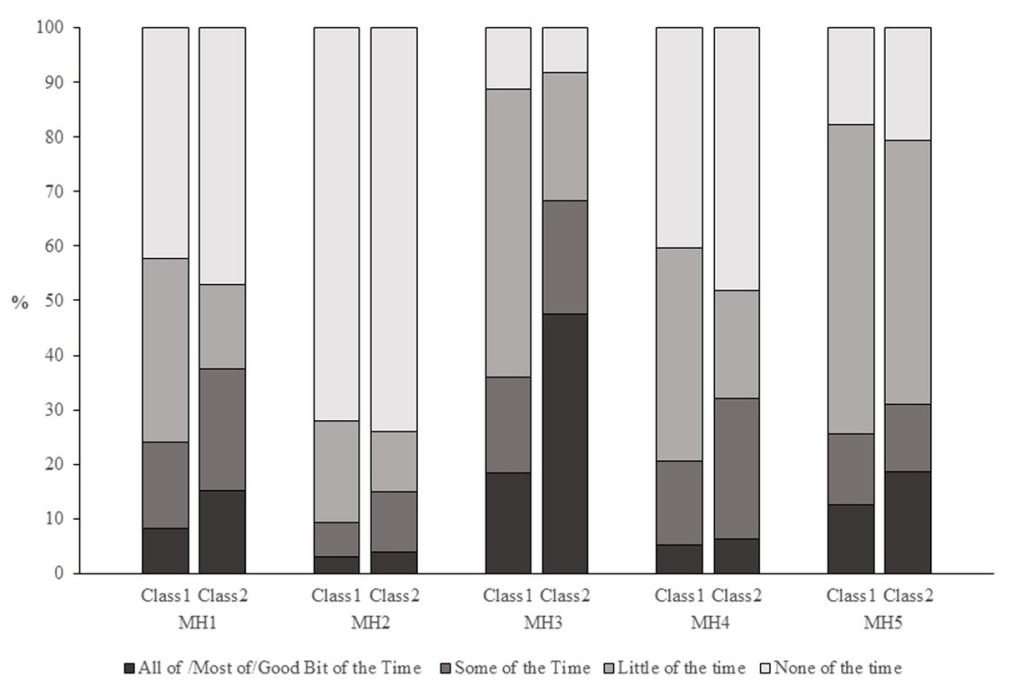

Fig. 2 Within-class item response percentages for the SF-36 mental health $(\mathrm{MH})$ sub-scale items. Legend: The selected best-fit latent variable mixture model had two classes. Response categories of "all of the time", "most of the time" and "good bit of the time" were combined due to low frequencies. MH3 and MH5 were reverse coded in the analysis

and the demographic and health status variables. For the three classes for the PF sub-scale items, the odds of class membership were higher for females, older respondents, those with a lower level of general health status, and lower HUI3 index scores in both of classes 2 and 3, relative to class 1. Respondents with lower educational attainment (i.e., less than high school), and who were overweight/ obese had a greater odds of being in class 3 than in class 1. For the MH sub-scale, the logistic model revealed that respondents of older age, with less than high school education, and good health had a greater odds of being in class 2 than class 1.

\section{Impact of DIF on predicted factor scores}

For the PF sub-scale, factor scores for the standard oneclass GRM and the three-class LVMM were strongly correlated (Pearson $r=0.89$ ). Despite the high correlation, there were differences in the tails of the distributions. The standardized difference was $\leq-0.5$ for $20.2 \%$ of the sample and $\geq 0.3$ for $26.2 \%$ of the sample (Fig. 3 ).

For the MH sub-scale, differences in factor scores between the one-class and two-class models for the $\mathrm{MH}$ items were also observed. Specifically, $2.6 \%$ of respondents had a standardized difference $\geq 0.3$ and $3.9 \%$ of respondents had a difference $\leq-0.3$ (Fig. 4).

There were differences in the CSEMs between the oneclass and multi-class models. For example, relative to the one-class model for the PF sub-scale items, in the threeclass model there was greater measurement precision in the lower range of the theta distribution (i.e., theta $<0$ ) for class 1 ; greater measurement precision was observed in the upper range of the theta distribution (theta $>0$ ) for class 3.

\section{Discussion}

Using a LVMM, this study identified three groups of individuals with different response patterns on the SF-36 PF sub-scale items and two groups of individuals with different response patterns on the $\mathrm{MH}$ sub-scale items in the population-based CaMos. For both sub-scale analyses, latent class 1 primarily contained respondents with fewer health limitations or problems. For the PF sub-scale, class 2 and 3 respondents were more likely to be female, older, and in poorer health than respondents in class 1 . For the $\mathrm{MH}$ sub-scale, older respondents with lower education and good health were more likely to be in class 2 . These findings indicate that DIF is present in unobserved groups, and the source of DIF can be at least partly explained by demographic and health status variables.

The results for the PF sub-scale are consistent with those from a previous study of the Canadian adult population [10], in which a three-class LVMM was found to fit the data better than one- or two-class models. We observed similar results in terms of item response patterns, effect of DIF on the latent factor scores, and the relationship between the latent class membership and selected socio-demographic and health variables. That previous study also found that the first latent class contained more people with no limitations in PF than the other two classes, and that sex, age and health status were associated with latent class membership. This provides support for our conclusion that DIF can affect responses to the SF-36 items and helps to validate the utility of the mixture model for exploring DIF.

For the MH sub-scale, we observed larger differences in both factor loadings and thresholds between the two latent classes for the following items: "Have you felt calm 
Table 4 Distribution (\%) and odds ratios (ORs) for demographic and health status characteristics by latent class

\begin{tabular}{|c|c|c|c|c|c|}
\hline & \multicolumn{3}{|c|}{ Distribution (\%) } & \multicolumn{2}{|l|}{ OR $(95 \% \mathrm{Cl})$} \\
\hline & Class 1 & Class 2 & Class 3 & Class 2 & Class 3 \\
\hline \multicolumn{6}{|l|}{ Physical Functioning } \\
\hline Class Frequencies & 5933 & 2081 & 1323 & & \\
\hline \multicolumn{6}{|l|}{ Sex } \\
\hline Men & 35.5 & 23.4 & 20.6 & Ref & Ref \\
\hline Women & 64.5 & 76.6 & 79.4 & $1.66(1.44,1.91)$ & $1.76(1.48,2.10)$ \\
\hline \multicolumn{6}{|l|}{ Age } \\
\hline 25-64 years & 60.5 & 40.7 & 31.0 & Ref & Ref \\
\hline$\geq 65$ years & 39.5 & 59.3 & 69.0 & $1.69(1.48,1.92)$ & $1.81(1.55,2.12)$ \\
\hline \multicolumn{6}{|l|}{ Education } \\
\hline Postsecondary & 53.7 & 43.7 & 33.0 & Ref & Ref \\
\hline High school graduate & 14.9 & 14.0 & 13.8 & $1.01(0.84,1.22)$ & $1.11(0.88,1.38)$ \\
\hline Less than high school & 31.4 & 42.4 & 53.2 & $1.13(0.98,1.30)$ & $1.32(1.12,1.56)$ \\
\hline \multicolumn{6}{|l|}{ General Health } \\
\hline Excellent/Very good & 68.1 & 42.0 & 30.0 & Ref & Ref \\
\hline Good & 26.3 & 43.2 & 40.7 & $1.99(1.73,2.29)$ & $\mathbf{1 . 8 6}(1.58,2.18)$ \\
\hline Fair/Poor & 5.6 & 14.8 & 29.3 & $2.42(1.94,3.01)$ & $\mathbf{3 . 5 3}(2.78,4.47)$ \\
\hline \multicolumn{6}{|l|}{ Weight Status } \\
\hline Underweight/Normal & 37.1 & 39.3 & 31.9 & Ref & Ref \\
\hline Overweight/Obese & 62.9 & 60.7 & 68.1 & $0.99(0.87,1.12)$ & $1.21(1.04,1.41)$ \\
\hline \multicolumn{6}{|l|}{ HUI3 } \\
\hline High $(\geq 0.8)$ & 81.1 & 58.9 & 38.3 & Ref & Ref \\
\hline Low $(<0.8)$ & 18.9 & 41.1 & 61.8 & $1.92(1.67,2.22)$ & $2.62(2.22,3.09)$ \\
\hline \multicolumn{6}{|l|}{ Mental Health } \\
\hline Class Frequencies & 7337 & 2058 & & & \\
\hline \multicolumn{6}{|l|}{ Sex } \\
\hline Men & 30.9 & 29.7 & & Ref & \\
\hline Women & 69.1 & 70.3 & & $1.03(0.90,1.17)$ & \\
\hline \multicolumn{6}{|l|}{ Age } \\
\hline 25-64 years & 53.8 & 44.8 & & Ref & \\
\hline$\geq 65$ years & 46.3 & 55.2 & & $\mathbf{1 . 1 8}(1.05,1.33)$ & \\
\hline \multicolumn{6}{|l|}{ Education } \\
\hline Postsecondary & 50.2 & 42.4 & & Ref & \\
\hline High school graduate & 14.6 & 14.1 & & $1.09(0.91,1.30)$ & \\
\hline Less than high school & 35.2 & 43.4 & & $1.21(1.06,1.38)$ & \\
\hline \multicolumn{6}{|l|}{ General Health } \\
\hline Excellent/Very good & 58.7 & 49.8 & & Ref & \\
\hline Good & 31.1 & 36.5 & & $1.13(1.00,1.28)$ & \\
\hline Fair/Poor & 10.3 & 13.8 & & $1.17(0.95,1.43)$ & \\
\hline
\end{tabular}


Table 4 Distribution (\%) and odds ratios (ORs) for demographic and health status characteristics by latent class (Continued)

\begin{tabular}{llll}
\hline Weight Status & & \\
Underweight/Normal & 37.4 & 34.8 & Ref \\
Overweight/Obese & 62.6 & 65.2 & $1.07(0.96,1.21)$ \\
HUI3 & & & Ref \\
High $(\geq 0.8)$ & 71.5 & 65.0 & $1.01(0.89,1.16)$ \\
Low $(<0.8)$ & 28.5 & 35.0 &
\end{tabular}

$95 \% \mathrm{Cl}=95 \%$ confidence interval; HUI3 = Health Utilities Index Mark 3; bold values indicate ORs that are statistically significant at $a=.05$. Class 1 is the reference group for both models. For the physical functioning sub-scale, the latent variable mixture model has three classes, while for the mental health sub-scale, the latent variable mixture model has two classes

and peaceful?", and "Have you been a happy person?". The low values of the factor loadings for the two items in the second latent class suggests that the two items have low ability to discriminate between respondents having higher and lower levels of mental health. In other words, a unit change in the factor scores in one class is not associated with the same change in the factor scores in the other class [5]. Possible reasons may be the different wording format for these two items when compared to the other $\mathrm{MH}$ items. Previous studies have reported that combinations of negatively and positively worded questions may compromise internal consistency of measures $[49,50]$.

The LVMM offers a number of advantages for DIF detection relative to traditional IRT models that focus on DIF investigations for manifest variables. The standard IRT model assumes there is one homogeneous group (i.e. one latent class) of individuals within a population and DIF is examined for subgroups of the population based on manifest characteristics. However, true sources of DIF may not be limited to manifest groups (i.e., sex, age) conditional on their latent ability. The LVMM is optimal for DIF examination when the origin(s) of differential response patterns is not known a priori. Prior studies have revealed that the mixture IRT model is a good alternative for detecting DIF $[15,17]$.

In implementing the LVMM, a critical step is to identify the best-fitting model with an appropriate number of latent classes. A potential problem can arise when the model converges on a local solution rather than the expected global maximum likelihood solution, resulting in biased model parameters [51]. To test the stability of the parameter estimates for the multiple-class models, we performed analyses by fitting models with the same number of classes but with different numbers of random starts [29]. The parameter estimates from the models with different sets of random starts were very similar, suggesting a stable and robust result. Another critical step is to characterize the latent classes. To do this, we used a pseudo-class methodology $[52,53]$ that has been shown to produce unbiased parameter estimates [52, 54]. Inaccurate numbers of latent classes may result from misspecification of the model [55], or the

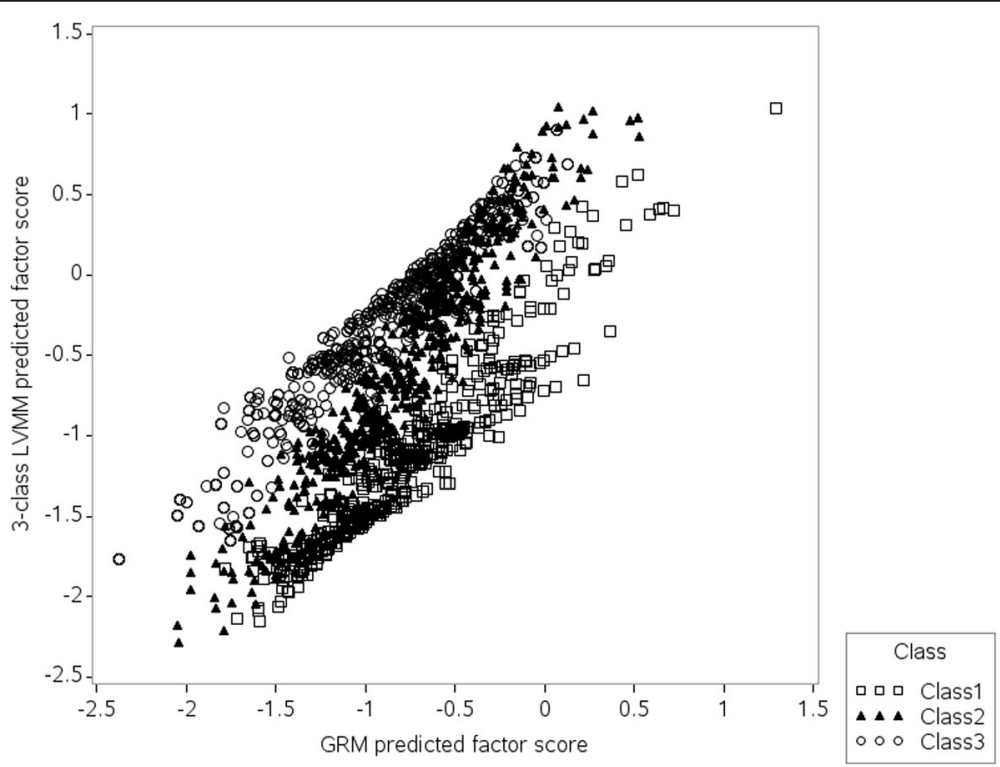

Fig. 3 Predicted factor scores for the SF-36 physical functioning (PF) sub-scale. Legend: Predicted factor scores were for the one-class graded response model (GRM) and the three-class latent variable mixture model (LVMM) 


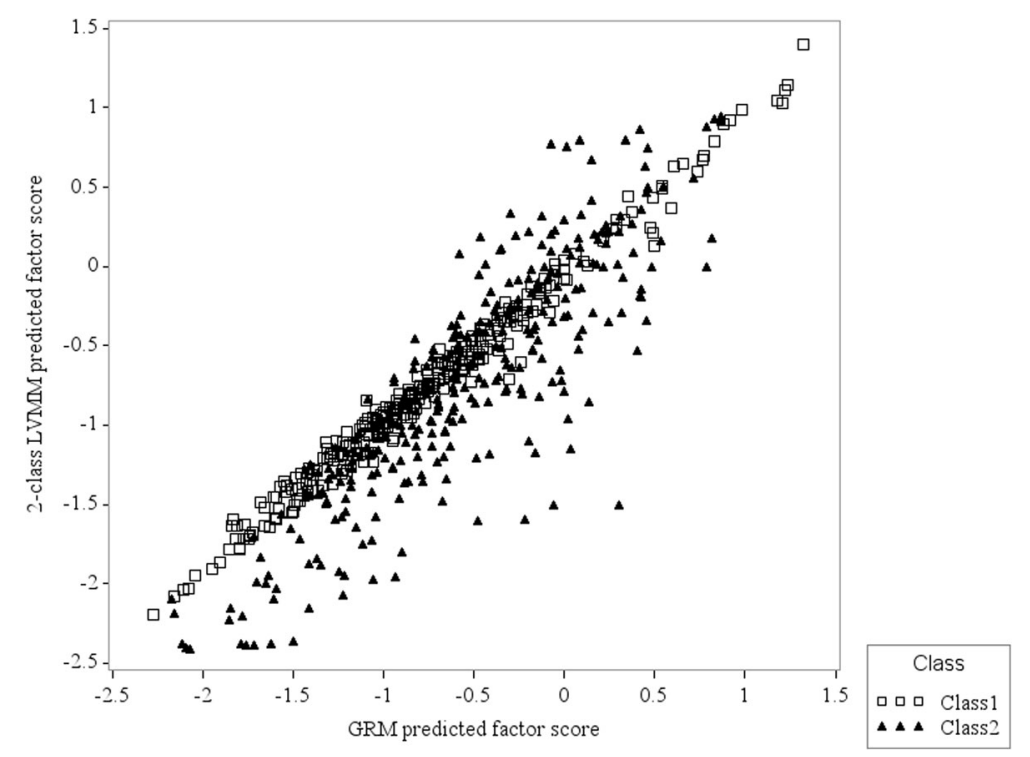

Fig. 4 Predicted factor scores for the SF-36 mental health (MH) sub-scale. Legend: Predicted factor scores were for the one-class graded response model (GRM) and the two-class latent variable mixture model (LVMM)

distribution of the data [56]. However, simulation and empirical studies have demonstrated the sensitivity of the LVMM [14-16, 20, 21, 57-59] for DIF assesment. Use of LVMMs can aid researchers to identify and characterize DIF effects in self-reported health outcome measures.

In the context of testing for DIF using the LVMM, there has been discussion in the literature about the optimal approach to scale the latent variable(s) in order to enable comparisons of item parameters. One approach is to allow latent factor means and variances to vary in each class, with equality constraints for item thresholds for one or more class-invariant items (also called anchor items) [60]. For this method, anchor items must be identified. Another approach is to set the latent factor means to zero and variances to one across classes; then the item parameters (i.e., factor loadings or item thresholds) are freely estimated across classes. We adopted this latter approach (i.e., with unequal item thresholds across classes). We subsequently tested for DIF on each item by fitting a set models with constrained item thresholds between latent classes for each item one at a time. The method has been used in previous studies for DIF detection using LVMMs $[20,61]$.

The study limitations must also be acknowledged. We analysed only the SF-36 PF and MH sub-scale items. Other sub-scales may also exhibit DIF, but they contain small numbers of items and therefore are more difficult to analyze using LVMMs. The sample was comprised of a smaller proportion of men and more older adults than in the general Canadian population; the findings may therefore not be representative of younger people and men. Although we observed comparable findings regarding DIF effects for the PF sub-scale with previous research in Canada [9], further research is needed to determine whether our findings are applicable to populations from other countries. Strengths of the present study include the use of a large national population-based sample, and the ability to consider both demographic and health status variables to characterize the latent classes. Other health status variables, such as the presence of selected chronic conditions, might be considered, if supported by research.

\section{Conclusions}

In conclusion, this study identified latent groups of respondents for whom the SF-36 PF and MH items function differently in a diverse national adult sample. The LVMM was a useful tool to define sub-groups of individuals with similar item response patterns and investigate characteristics potentially associated with group member. This analysis provides information that can be useful for generating hypotheses for future studies about DIF; for example, one might use these results to conduct DIF analyses for observed population sub-groups in independent samples. Testing for DIF should be a routine part of comparative analyses of population health status. The comparability of SF-36 sub-scale scores can be significantly compromised by heterogeneity in item responses, which can affect the interpretation of results from clinical and epidemiologic studies. Future study is needed to validate the present findings in other samples to inform generalizability of the results. Future research might also compare the sensitivity of the PF and $\mathrm{MH}$ sub-scales to detect DIF effects using both manifest variable and LVMM approaches. 


\section{Appendix}

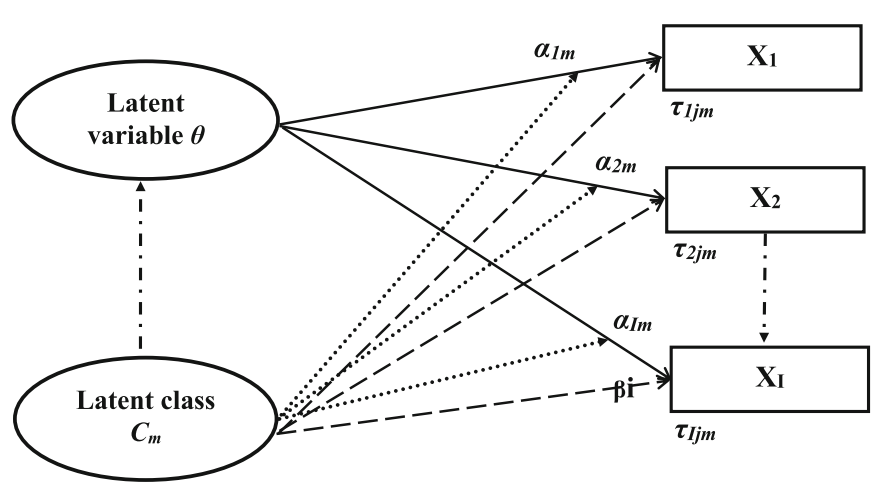

Factor loadings $\left(\boldsymbol{\alpha}_{i} ; i=1, \ldots, I\right)$ for items $\mathbf{X}_{1}$ to $\mathbf{X}_{I}$ vary across the $m$ classes $(m=1, \ldots, M)$

$-\rightarrow$ Thresholds $(\boldsymbol{\tau})$ for $j-1$ response categories per item vary across the $m$ classes

$-\cdot \rightarrow$ Variance of the latent factor $(\theta)$ may vary between the latent classes

Fig. 5 The latent variable mixture model (LVMM) with class-specific parameters

\section{Acknowledgements}

We thank all those participants in CaMos whose careful responses and attendance made this analysis possible. We thank the CaMos Research Group for access to the data to complete this study:

- David Goltzman (co-principal investigator, McGill University), Nancy Kreiger (co-principal investigator, Toronto), Alan Tenenhouse (principal investigator emeritus, Toronto),

- CaMos Coordinating Centre, McGill University, Montreal, Quebec: Suzette Poliquin (national coordinator emeritus), Suzanne Godmaire (research assistant), Silvia Dumont (administrative assistant), Claudie Berger (study statistician), Lisa Langsetmo (Fellow).

- Memorial University, St. John's, Newfoundland: Carol Joyce (director), Christopher Kovacs (co-director), Emma Sheppard (coordinator).

- Dalhousie University, Halifax, Nova Scotia: Susan Kirkland, Stephanie Kaiser (co-directors), Barbara Stanfield (coordinator).

- Laval University, Quebec City, Quebec: Jacques P. Brown (director), Louis Bessette (co-director), Marc Gendreau (coordinator).

- Queen's University, Kingston, Ontario: Tassos Anastassiades (director), Tanveer Towheed (co-director), Barbara Matthews (coordinator), Wilma Hopman (scientist).

- University of Toronto, Toronto, Ontario: Bob Josse (director), Sophie Jamal (co-director), Tim Murray (past director), Barbara Gardner-Bray (coordinator), Angela Cheung (scientist).

- McMaster University, Hamilton, Ontario: Jonathan D. Adachi (director), Alexandra Papaioannou (co-director), Laura Pickard (coordinator).

- University of Saskatchewan, Saskatoon, Saskatchewan: Wojciech P. Olszynski (director), K. Shawn Davison (co-director), Jola Thingvold (coordinator). - University of Calgary, Calgary, Alberta: David A. Hanley (director), Jane Allan (coordinator).

- University of British Columbia, Vancouver, British Columbia: Jerilynn C. Prior (director), Millan Patel (co-director), Nera Andjelic (coordinator), Brian Lentle (radiologist)

\section{Funding}

The research team was funded by the Canadian Institutes of Health Research (Funding Reference \#122110). LML is supported by a Manitoba Research Chair from Research Manitoba. CaMos is currently supported by the Canadian Institutes of Health Research, Amgen Canada Inc., Dairy Farmers of Canada, Merck Canada, Eli Lilly Canada, and Novartis Canada.

\section{Availability of data and materials}

The data that support the findings of this study are available from the CaMos principal investigators, Dr. David Goltzman and Dr. Nancy Krieger, but restricions apply to the availability of these data. The CaMos has developed an Ancillary Project and Data Release Policy that governs ancillary project approval and access to the data. To obtain a copy of the Ancillary Project and Data Release Policy and the requirements for proposal submission, or to obtain further information from the CaMos principal investigators about data access, please send an e-mail to info@camos.org.

\section{Authors' contributions}

$X W, R S, W H, N M, T T S, J L$, and $L M L$ conceived the study design. WH, JP, AP, $R G J, T$, and KSD contributed to acquisition of the data and interpretation of study results. XW, RS, and $L M L$ analyzed the data. XW, RS, and $L M L$ drafted the manuscript. All authors provided feedback on a draft version of the manuscript and read and approved the final manuscript.

\section{Competing interests}

The authors declare that they have no competing interests.

\section{Consent for publication}

Not applicable.

\section{Ethics approval and consent to participate}

Approval for this research was provided by the University of Manitoba Health Research Ethics Board. All study particiants provided consent to participate in the study.

\section{Publisher's Note}

Springer Nature remains neutral with regard to jurisdictional claims in published maps and institutional affiliations.

\section{Author details}

${ }^{1}$ School of Public Health, University of Alberta, Edmonton, AB, Canada. ${ }^{2}$ School of Public Health and Health Management, Weifang Medical University, Weifang, Shandong Province, China. ${ }^{3}$ School of Nursing, Trinity Western University \& Centre for Health Evaluation and Outcomes Sciences, Providence Health Care, Langley, BC, Canada. ${ }^{4}$ Department of Public Health Sciences, Queen's University, Kingston, ON, Canada. ${ }^{5} \mathrm{McGill}$ University Health Centre, Montréal, QC, Canada. ${ }^{6}$ Department of Community Health Sciences \& 
O'Brien Institute for Public Health, University of Calgary, Calgary, AB, Canada. ${ }^{7}$ Department of Mathematics and Statistics, University of Saskatchewan, Saskatoon, SK, Canada. ${ }^{8}$ Division of Endocrinology, Department of Medicine, University of British Columbia, Vancouver, BC, Canada. ${ }^{9}$ Department of Medicine, McMaster University, Hamilton, ON, Canada. ${ }^{10}$ Department of Medicine, Faculty of Medicine, University of Toronto, Toronto, ON, Canada. ${ }^{11}$ Department of Medicine, Queen's University, Kingston, ON, Canada. ${ }^{12}$ Faculty of Graduate Studies, University of Victoria, Victoria, BC, Canada. ${ }^{13}$ Department of Community Health Sciences, University of Manitoba, S113-750 Bannatyne Ave, Winnipeg, MB R3E OW3, Canada.

\section{Received: 11 July 2016 Accepted: 4 May 2017}

\section{Published online: 15 May 2017}

\section{References}

1. Hopman WM, Towheed T, Anastassiades T, Tenenhouse A, Poliquin S, Berger C, et al. Canadian normative data for the SF-36 health survey. Canadian Multicentre osteoporosis study research group. Can Med Assoc J. 2000;163:265-71.

2. Reeve BB, Wyrwich KW, Wu AW, Velikova G, Terwee CB, Snyder CF, et al. ISOQOL recommends minimum standards for patient-reported outcome measures used in patient-centered outcomes and comparative effectiveness research. Qual Life Res. 2013;22:1889-905.

3. Mokkink LB, Terwee CB, Patrick DL, Alonso J, Stratford PW, Knol DL, et al. The COSMIN checklist for assessing the methodological quality of studies on measurement properties of health status measurement instruments: an international Delphi study. Qual Life Res. 2010;19:539-49.

4. Terwee CB, Bot SD, de Boer MR, van der Windt DA, Knol DL, Dekker J, et al. Quality criteria were proposed for measurement properties of health status questionnaires. J Clin Epidemiol. 2007;60:34-42.

5. Lix LM, Acan Osman B, Adachi JD, Towheed T, Hopman W, Davison KS, Leslie WD. Measurement equivalence of the SF-36 in the Canadian Multicentre osteoporosis study. Health Qual Life Outcomes. 2012;10:29.

6. Vandenberg R, Lance C. A review and synthesis of the measurement invariance literature: suggestions, practices, and recommendations for organizational research. Organ Res Methods. 2000;3(1):4-70.

7. Teresi JA, Fleishman JA. Differential item functioning and health assessment. Qual Life Res. 2007;16(Suppl 1):33-42.

8. Lix LM, Wu X, Hopman W, Mayo N, Sajobi TT, Liu J, et al. Differential item functioning in the SF-36 physical functioning and mental health sub-scales: a population-based investigation in the Canadian Multicentre osteoporosis study. PLoS One. 2016;11(3):e0151519.

9. Perkins AJ, Stump TE, Monahan PO, McHorney CA. Assessment of differential item functioning for demographic comparisons in the MOS SF36 health survey. Qual Life Res. 2006;15:331-48.

10. Sawatzky R, Ratner PA, Kopec JA, Zumbo BD. Latent variable mixture models: a promising approach for the validation of patient reported outcomes. Qual Life Res. 2012;21:637-50.

11. Bjorner JB, Kreiner S, Ware JE, Damsgaard MT, Bech P. Differential item functioning in the Danish translation of the SF-36. J Clin Epidemiol. 1998;51: 1189-202.

12. Fleishman JA, Lawrence WF. Demographic variation in SF-12 scores: true differences or differential item functioning? Med Care. 2003;41(7 Suppl): III75-86.

13. Edelen $\mathrm{MO}$, Reeve BB. Applying item response theory (IRT) modeling to questionnaire development, evaluation, and refinement. Qual Life Res. 2007; 16(Suppl 1):5-18.

14. Oliveri ME, Ercikan K, Zumbo B. Analysis of sources of latent class differential item functioning in international assessments. Int J Test. 2013;13:272-93.

15. Maij-de Meij AM, Kelderman $\mathrm{H}$, van der Flier $\mathrm{H}$. Improvement in detection of differential item functioning using a mixture item response theory model. Multivar Behav Res. 2010;45:975-99.

16. Cohen AS, Bolt DM. A mixture model analysis of differential item functioning. J Educ Meas. 2005;42:133-48.

17. Samuelsen KM. Examining differential item functioning from a latent class perspective. In G. R. Hancock \& K. M. Samuelsen, editors. Advances in latent variable mixture models, Charlotte NC: Information Age Publishing; 2008, p. 177-197.

18. Lubke G, Neale M. Distinguishing between latent classes and continuous factors with categorical outcomes: class invariance of parameters of factor mixture models. Multivar Behav Res. 2008;43:592-620.
19. De Ayala RJ, Kim S, Stapleton LM, Dayton CM. Differential item functioning: a mixture distribution conceptualization. Int J Test. 2002;2:243-76.

20. Chen $Y$, Jiao H. Exploring the utility of background and cognitive variables in explaining latent differential item functioning: an example of the PISA 2009 reading assessment. Educ Assess. 2014;19:77-96.

21. Webb ML, Cohen AS, Schwanenflugel PJ. Latent class analysis of differential item functioning on the Peabody picture vocabulary test-III. Educ Psychol Meas. 2008;68:335-51.

22. Yu YF, Yu AP, Ahn J. Investigating differential item functioning by chronic diseases in the SF-36 health survey: a latent trait analysis using MIMIC models. Med Care. 2007:45:851-9.

23. Pollard B, Johnston M, Dixon D. Exploring differential item functioning in the SF-36 by demographic, clinical, psychological and social factors in an osteoarthritis population. BMC Musculoskelet Disord. 2013;14:346.

24. Kreiger N, Tenenhouse A, Joseph L, Mackenzie T, Poliquin S, Brown JP, et al. The Canadian Multicentre osteoporosis study (CaMos): background, rationale, methods. Can J Aging. 1999;18:376-87.

25. Ware JE Jr, Sherbourne CD. The MOS 36-item short-form health survey (SF36). I. Conceptual framework and item selection. Med Care. 1992;30:473-83.

26. Horsman J, Furlong W, Feeny D, Torrance G. The health utilities index (HUI): concepts, measurement properties and applications. Health Qual Life Outcomes. 2003;1:54.

27. Feeny D, Furlong W, Torrance GW, Goldsmith $\mathrm{CH}$, Zhu Z, DePauw S, et al. Multi-attribute and single-attribute utility functions for the health utilities index mark 3 system. Med Care. 2002;40:113-28.

28. Haberman SJ. The analysis of residuals in cross-classified tables. Biometrics. 1973;29:205-20.

29. Muthén LK, Muthén BO. Statistical analysis with latent variables. MPlus user guide, 7th ed. 2012. Los Angeles, CA: Muthén \& Muthén.

30. MacCallum RC, Browne MW, Sugawara HM. Power analysis and determination of sample size for covariance structure modeling. Psychol Methods. 1996;1:130-49.

31. Hu LT, Bentler PM. Cutoff criteria for fit indices in covariance structure analysis: conventional criteria versus new alternatives. Struct Equ Model. 1999;6:1-55

32. Chen W, Thissen D. Local dependence indexes for item pairs using item response theory. J Educ Behav Stat. 1997;22:265-89.

33. Samejima, F. (1969). Estimation of latent ability using a response pattern of graded scores. Psychometrika. 1969 34:100.

34. Muthén B. Latent variables hybrids: overview of old and new models. In G. R. Hancock \& K. M. Samuelsen, editors, Advances in latent variable mixture models (pp. 1-24). 2008. Charlotte, NC: Information Age Publishing.

35. Kamata A, Bauer DJ. A note on the relation between factor analytic and item response theory models. Struct Equ Model. 2008;15:136-53.

36. Dayton C. Latent class scaling analysis. Thousand Oaks, CA: Sage; 1998.

37. Nylund KL, Asparouhov T, Muthén BO. Deciding on the number of classes in latent class analysis and growth mixture modeling: a Monte Carlo simulation study. Struct Equ Model. 2007;14:535-69.

38. Li F, Cohen AS, Kim S, Cho S. Model selection methods for mixture dichotomous IRT models. Appl Psychol Meas. 2009;33:353-73.

39. Vuong $\mathrm{QH}$. Likelihood ratio tests for model selection and non-nested hypotheses. Econometrica. 1989;57:307-33.

40. Muthén B, Brown CH, Masyn K, Jo B, Khoo ST, Yang CC, et al. General growth mixture modeling for randomized preventive interventions. Biostatistics. 2002;3:459-75.

41. Holland PW. A framework and history for score linking. In: Dorans NJ, Pommerich M, Holland PW, editors. Linking and aligning scores and scales. New York: Springer; 2007. p. 5-30.

42. Kolen MJ, Brennan RL. Test equating, scaling, and linking. Methods and practices. 2nd ed. New York: Springer; 2004.

43. Cohen AS, Kim S, Wollack JA. An investigation of the likelihood ratio test for detection of differential item functioning. Appl Psychol Meas. 1996;20:15-26.

44. Canada H. Canadian guidelines for body weight classification in adults (publication ID 4645). Ottawa: Health Canada Publications Centre; 2003.

45. De Ayala RJ. The theory and practice of item response theory. New York: Guilford Press; 2009.

46. Joanes DN, Gill CA. Comparing measures of sample skewness and kurtosis. Statistician. 1998:47:183-9.

47. SAS Institute Inc. SAS/STAT 9.3 User's guide. Cary, NC: SAS Institute Inc; 2013. 
48. Reise SP, Gomel JN. Modeling qualitative variation within latent trait dimensions: application of mixed-measurement to personality assessment. Multivar Behav Res. 1995;30:341-58.

49. Spector PE, Van Katwyk PT, Brannick MT, Chen PY. When two factors don't reflect two constructs: how item characteristics can produce artifactual factors. J Manag. 1997;23:659-77.

50. Sawatzky R, Ratner PA, Johnson JL, Kopec JA, Zumbo BD. Sample heterogeneity and the measurement structure of the multidimensional students' life satisfaction scale. SOCl. 2009;94:273-96.

51. Rost J. A logistic mixture distribution model for polychotomous item responses. Br J Math Stat Psychol. 1991;44:75-92.

52. Wang CP, Hendricks BC, Bandeen-Roche K. Residual diagnostics for growth mixture models: examining the impact of a preventive intervention on multiple trajectories of aggressive behavior. JAMA. 2005;100:1054-76.

53. Muthén $B$, Muthén $L$. Wald test of mean equality for potential latent class predictors in mixture modeling. 2007. http://www.statmodel.com/ download/MeanTest1.pdf. Accessed 30 Mar, 2016.

54. Clark SL, Muthén B. Relating latent class analysis results to variables not included in the analysis. 2009. http://www.statmodel.com/download/ relatinglca.pdf. Accessed 30 Mar, 2016.

55. Bauer DJ, Curran PJ. The integration of continuous and discrete latent variable models: potential problems and promising opportunities. Psychol Methods. 2004;9:3-29.

56. Bauer DJ, Curran PJ. Distributional assumptions of growth mixture models: implications for over extraction of latent trajectory classes. Psychol Methods. 2003;8:338-63.

57. Lubke $\mathrm{G}$, Muthén BO. Performance of factor mixture models as a function of model size, covariate effects, and class-specific parameters. Struct Equ Model. 2007;14:26-47.

58. Finch $\mathrm{WH}$, French BF. Parameter estimation with mixture item response theory models: a Monte Carlo comparison of maximum likelihood and Bayesian methods. J Mod App Stat Methods. 2012;11:167-78.

59. Jiao H, Macready G, Liu J, Cho Y. A mixture Rasch model-based computerized adaptive test for latent class identification. Appl Psychol Meas. 2012;36:469-93.

60. Paek I, Cho S-J. A note on parameter estimate comparability: across latent classes in mixture IRT modeling. Appl Psychol Meas. 2015;39(2):135-43.

61. DeMars CE, Lau A. Differential item functioning detection with latent classes: how accurately can we detect who is responding differentially? Ed Psychol Meas. 2011;4:597-616.

\section{Submit your next manuscript to BioMed Central and we will help you at every step:}

- We accept pre-submission inquiries

- Our selector tool helps you to find the most relevant journal

- We provide round the clock customer support

- Convenient online submission

- Thorough peer review

- Inclusion in PubMed and all major indexing services

- Maximum visibility for your research

Submit your manuscript at www.biomedcentral.com/submit 